\title{
DSCC2019-8960
}

\section{LANE KEEPING CONTROL USING FINITE SPECTRUM ASSIGNMENT WITH MODELING ERRORS}

\author{
Illés Vörös \\ Department of Applied Mechanics \\ Budapest University of Technology \\ and Economics \\ Budapest, $\mathrm{H}-1111$ \\ Hungary \\ Email: illes.voros@mm.bme.hu
}

\author{
Balázs Várszegi \\ Department of Applied Mechanics \\ Budapest University of Technology \\ and Economics \\ Budapest, $\mathrm{H}-1111$ \\ Hungary \\ varszegi@mm.bme.hu
}

\author{
Dénes Takács \\ MTA-BME Research Group on \\ Dynamics of Machines and Vehicles \\ Budapest, $\mathrm{H}-1111$ \\ Hungary \\ takacs@mm.bme.hu
}

\begin{abstract}
Lane keeping control of the single track vehicle model with linear tire characteristics is analyzed in the presence of time delay. In order to compensate time delay, the predictor control approach called finite spectrum assignment is applied. This controller uses an internal model of the plant to predict current system states in spite of the time delay. The predictions are based on a simplified version of the vehicle model, neglecting tire dynamics. The predictive control approach is compared with traditional feedback control using analytically derived stability maps and numerical simulations. Robustness to parameter mismatches and numerical issues related to the implementation of the control law are also analyzed.
\end{abstract}

\section{INTRODUCTION}

One of the fundamental problems related to a variety of advanced driver assistance systems and autonomous driving in general is the lateral positioning of the vehicle. Be it a lane change maneuver, lane keeping assistance or general path following, it is important to have reliable control of the vehicle's lateral position.

It is therefore not surprising that a large number of approaches have been proposed over the years [1]. Kinematic controllers, such as pure pursuit [2] or the Stanley controller [3] are the most common solutions because of their simplicity. These controllers can be extended by the consideration of the dynamic effects acting on the vehicle [4]. Another popular approach is optimal control (especially the linear quadratic regulator), where a certain cost function is specified, and the control gains are chosen so that this cost function is minimized [5]. Model predictive control is also widely covered in the automotive literature [6]. These controllers find the best control input that optimizes the system response according to a plant model and a cost function. Other solutions include adaptive, and classical, such as PID or sliding mode control. For more details, see the overview of control methods in [1] and the references therein.

In the analysis of these controllers, the presence of time delay is rarely considered, although it can influence control performance, see [7]. The overall time delay may originate from sensor delays, communication and computation times, as well as non modeled actuator dynamics. Position estimation, in particular, may contribute a large part to the overall delay of the system, especially when vision-based approaches are involved. The corresponding image processing algorithms are generally rather resource intensive, and most commercialized solutions are not capable of processing road information with high frequency $[8,9]$. As an example, the vision delay was considered to amount to $0.15 \mathrm{~s}$ in [10] and $0.2 \mathrm{~s}$ in [11]. In [12], a time constant of $0.3 \mathrm{~s}$ was used for a low level steering angle controller, while [3] used $0.4 \mathrm{~s}$ as a conservative estimate for the steering delay.

In order to compensate time delay, the predictor control approach called finite spectrum assignment (FSA) is going to be 
used in this paper [13-15]. Similarly to the classical Smith predictor, this controller uses a mathematical model of the plant to calculate predictions of the current system states. Thus instead of the delayed feedback signals, these predictions can be used to control the system. In the ideal case, if the mathematical model in the controller perfectly matches the real system, the control signal is implemented accurately and there are no disturbances or measurement noise, then the FSA controller cancels out the delay terms, and the predicted states will be equal to the actual system states.

This paper is a continuation of our previous work [16], where we demonstrated the use of the FSA controller through a simple vehicle model. In [16], the internal model of the controller was a perfect representation of the controlled system, and modeling errors could only stem from parameter mismatches. In this paper, we extend the model of the controlled vehicle with the inclusion of tire side slip, which allows the dynamics of the internal model to diverge from the controlled system, similarly to real life applications. Thus, the dynamics of the two models are different, but both models are simple enough to allow analytical calculations. In addition, the effects of parameter mismatches between the models in terms of vehicle longitudinal velocity and time delay are also analyzed using stability maps and numerical simulations. Since the emphasis in our analysis is on the effects of time delay and modeling errors, we are only going to consider a simple straight-line trajectory. As this is an equilibrium state of the system, the corresponding stability criteria can be derived in a straightforward, analytical manner.

The rest of the paper is organized as follows: first, the equations of motion for both the kinematic and the dynamic vehicle models are derived. Next, the stability analysis of delayed state feedback is presented. Finite spectrum assignment is detailed afterwards, including a theoretical overview and its application to the dynamic vehicle model. In the last section, instability mechanisms related to the implementation of the control law and the effects of parameter mismatches in the model are demonstrated.

\section{VEHICLE MODELS}

Two versions of the single-track vehicle model $[17,18]$ are presented in this section. The simpler, kinematic model is used within the controller as the base of predictions, while the dynamic vehicle model (which includes lateral tire dynamics) forms the controlled system.

\section{Kinematic Vehicle Model}

In this simplified form of the bicycle model (see Fig. 1 (a)), we assume that no tire side slip occurs at the wheels and the longitudinal velocity $V$ of the vehicle is kept constant. The resulting model is very compact, it only includes a small number of parameters and the computational cost of its numerical integration

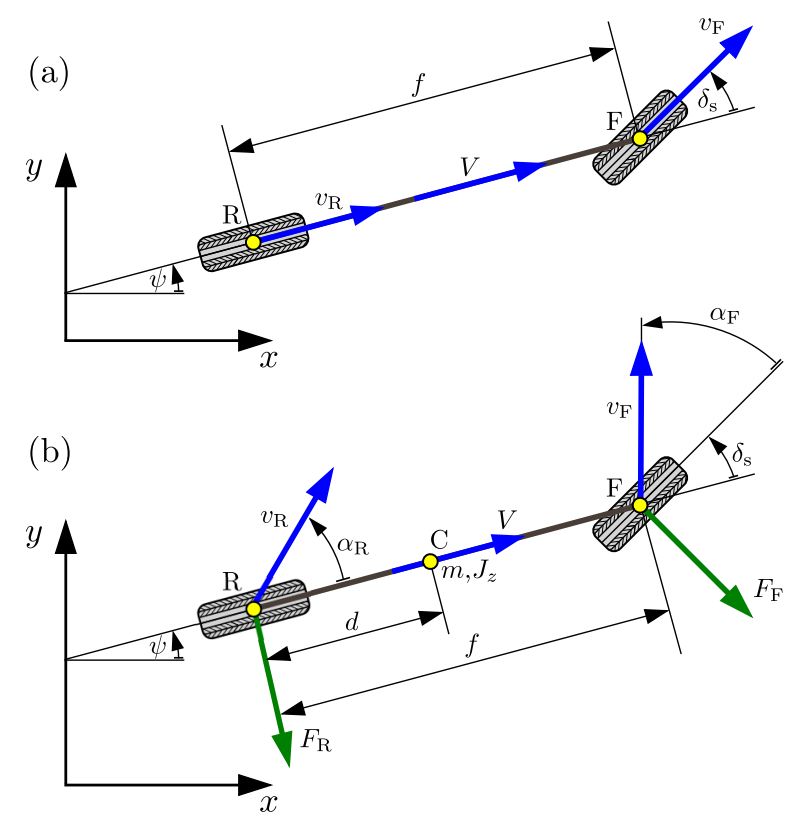

FIGURE 1. The kinematic (a) and dynamic (b) vehicle models [17, $18]$.

is low, which makes it ideal for control purposes.

The assumption of zero tire side slip forces the velocity vectors at the front and rear axles (represented by points $\mathrm{F}$ and $\mathrm{R}$ ) to be parallel with the planes of the wheels. This can be described using kinematic constraints. In addition, the assumption of a constant longitudinal velocity adds another kinematic constraint. The resulting constraint equations are

$$
\begin{aligned}
\dot{x} \sin \left(\psi+\delta_{\mathrm{s}}\right)-\dot{y} \cos \left(\psi+\delta_{\mathrm{s}}\right)-f \dot{\psi} \cos \delta_{\mathrm{s}} & =0, \\
-\dot{x} \sin \psi+\dot{y} \cos \psi & =0, \\
\dot{x} \cos \psi+\dot{y} \sin \psi & =V,
\end{aligned}
$$

where the system states are the coordinates $x$ and $y$ of the rear axle (point $\mathrm{R}$ ) and the vehicle heading $\psi$. The steering angle, which is going to be used as control input, is denoted by $\delta_{\mathrm{s}}$, and the wheelbase is $f$. The equations of motion can be reached by solving the above constraint equations for the state derivatives:

$$
\begin{aligned}
\dot{x}(t) & =V \cos \psi(t), \\
\dot{y}(t) & =V \sin \psi(t), \\
\dot{\psi}(t) & =\frac{V}{f} \tan \delta_{\mathrm{s}}(t) .
\end{aligned}
$$

\section{Dynamic Vehicle Model}

The main difference to the previous model is that tire side slip may occur in the dynamic vehicle model, which means the 
velocity vectors of points $\mathrm{F}$ and $\mathrm{R}$ do not necessarily point in the same direction as the wheels (see Fig. 1 (b)).

In order to derive the equations of motion, we keep only one of the three kinematic constraints of the previous model, namely, the longitudinal speed of the vehicle is still assumed to be constant. The equations of motion can be derived by means of the Lagrange-d'Alembert method [19], or by more efficient alternatives like the Gibbs-Appell [20-22] or Kane's method [21,23]. Here, we are going to apply the Gibbs-Appell method, which is commonly used for low degrees-of-freedom nonholonomic systems $[24,25]$. One great advantage of this method is that the states can be handled in a ground-fixed coordinate system, while the velocities can be described in a body-fixed one.

For the derivation of the equations of motion, 2 pseudo velocities must be chosen, since the difference between the number of states (3: $x, y, \psi)$ and the kinematic constraints (1: longitudinal speed of the vehicle is assumed to be constant) is 2 . Although the pseudo velocities are chosen intuitively, they must satisfy the kinematic constraints. In our case, $\sigma_{1}$ is the lateral velocity of point $R$ of the vehicle, while $\sigma_{2}$ is the yaw rate. The definition of the pseudo velocities along with the kinematic constraint results in a system of three equations, from which the state derivatives can be expressed as:

$$
\begin{aligned}
\dot{x} & =V \cos \psi-\sigma_{1} \sin \psi, \\
\dot{y} & =V \sin \psi+\sigma_{1} \cos \psi, \\
\dot{\psi} & =\sigma_{2} .
\end{aligned}
$$

The equations for the pseudo-accelerations, that define the dynamics of the system, can be reached using the Gibbs-Appell equations

$$
\frac{\partial G}{\partial \dot{\sigma}_{i}}=\Gamma_{i}, \quad i=1,2,
$$

where $G$ is the so-called energy of acceleration (or Gibbs function) that is defined as

$$
G=\frac{1}{2} m a_{\mathrm{C}}^{2}+\frac{1}{2} J_{z} \ddot{\Psi}^{2}+\ldots
$$

for in-plane motion. Here $a_{\mathrm{C}}$ is the acceleration of the center of gravity and $\ddot{\psi}$ is the angular acceleration of the vehicle. The energy of acceleration includes further terms that disappear after performing the derivation in Eqn. (4). Expressing $a_{\mathrm{C}}$ and $\ddot{\psi}$ as a function of the pseudo-velocities and their time derivatives, the derivations result in

$$
\begin{aligned}
& \frac{\partial G}{\partial \dot{\sigma}_{1}}=m\left(d \dot{\sigma}_{2}+\dot{\sigma}_{1}+V \sigma_{2}\right) \\
& \frac{\partial G}{\partial \dot{\sigma}_{2}}=\left(d^{2} m+J_{z}\right) \dot{\sigma}_{2}+d m\left(\dot{\sigma}_{1}+V \sigma_{2}\right) .
\end{aligned}
$$

The right-hand side of Eqn. (4) includes the pseudo-forces $\Gamma_{i}$ that can be determined from the virtual power of the active forces. In our model, active forces emerge at the tires, where we consider the side slip forces as

$$
F_{i}^{\text {lat }}=C_{i} \alpha_{i}, \quad i \in\{\mathrm{F}, \mathrm{R}\},
$$

where $C_{i}$ refers to the cornering stiffness and the indeces $\mathrm{F}$ and $\mathrm{R}$ denote the front and rear wheels respectively.

Since the resultant lateral force $F^{\text {lat }}$ is not applied at the center of the contact patch, it also generates an aligning torque. This, however, is neglected in our analysis, because the corresponding moment arm is insignificant compared to the vehicle geometry.

The slip angles in our case are

$$
\begin{aligned}
& \alpha_{\mathrm{F}}=\arctan \left(\frac{\dot{y}+f \cos \psi \dot{\psi}}{\dot{x}-f \sin \psi \dot{\psi}}\right)-\psi-\delta_{\mathrm{s}} \\
& \alpha_{\mathrm{R}}=\arctan \left(\frac{\dot{y}}{\dot{x}}\right)-\psi
\end{aligned}
$$

Transforming the tire forces into the global coordinate system leads to

$$
\begin{aligned}
& \mathbf{F}_{\mathrm{F}}=\left[\begin{array}{c}
F_{\mathrm{F}, x} \\
F_{\mathrm{F}, y} \\
0
\end{array}\right]=\left[\begin{array}{c}
F_{\mathrm{F}}^{\text {lat }} \sin \left(\psi+\delta_{\mathrm{s}}\right) \\
-F_{\mathrm{F}}^{l a t} \cos \left(\psi+\delta_{\mathrm{s}}\right) \\
0
\end{array}\right], \\
& \mathbf{F}_{\mathrm{R}}=\left[\begin{array}{c}
F_{\mathrm{R}, x} \\
F_{\mathrm{R}, y} \\
0
\end{array}\right]=\left[\begin{array}{c}
F_{\mathrm{R}}^{\text {lat } \sin \psi} \\
-F_{\mathrm{R}}^{l a t} \cos \psi \\
0
\end{array}\right] .
\end{aligned}
$$

This way the virtual power is

$$
\delta P=\mathbf{F}_{\mathrm{F}} \delta \mathbf{v}_{\mathrm{F}}+\mathbf{F}_{\mathrm{R}} \delta \mathbf{v}_{\mathrm{R}},
$$

where $\delta$ denotes the virtual quantities. Expanding Eqn. (12) gives

$$
\begin{aligned}
\delta P & =\delta \sigma_{1}\left(\left(F_{\mathrm{F}, y}+F_{\mathrm{R}, y}\right) \cos \psi-\left(F_{\mathrm{F}, x}+F_{\mathrm{R}, x}\right) \sin \psi\right)+ \\
& +\delta \sigma_{2} f\left(F_{\mathrm{F}, y} \cos \psi-F_{\mathrm{F}, x} \sin \psi\right),
\end{aligned}
$$


where the notations $F_{i, j}$ represent the $x$ or $y$ components of the lateral force vectors in Eqn. (11). The coefficients of the virtual pseudo-velocities in Eqn. (13) form the pseudo-forces:

$$
\begin{aligned}
& \Gamma_{1}=\left(F_{\mathrm{F}, y}+F_{\mathrm{R}, y}\right) \cos \psi-\left(F_{\mathrm{F}, x}+F_{\mathrm{R}, x}\right) \sin \psi, \\
& \Gamma_{2}=f\left(F_{\mathrm{F}, y} \cos \psi-F_{\mathrm{F}, x} \sin \psi\right) .
\end{aligned}
$$

Using Eqn. (6) with Eqn. (14) and Eqn. (7) with Eqn. (15), the equations of motion can be given as

$$
\begin{gathered}
m\left(\dot{\sigma}_{1}-V \sigma_{2}\right)=-\frac{d^{2} m+J_{z}}{J_{z}}\left(\sin \psi\left(F_{\mathrm{F}, x}+F_{\mathrm{R}, x}\right)\right. \\
\left.-\cos \psi\left(F_{\mathrm{F}, y}+F_{\mathrm{R}, y}\right)\right)+\frac{f d m}{J_{z}}\left(\sin \psi F_{\mathrm{F}, x}-\cos \psi F_{\mathrm{F}, y}\right) \\
J_{z} \dot{\sigma}_{2}=\sin \psi\left((d-f) F_{\mathrm{F}, x}+d F_{\mathrm{R}, x}\right)-\cos \psi\left((d-f) F_{\mathrm{F}, y}+d F_{\mathrm{R}, y}\right)
\end{gathered}
$$

The above two equations (after substituting the force components according to Eqn. (11)), along with the general coordinate velocities in Eqn. (3) form the governing equations of the dynamic vehicle model.

\section{FEEDBACK CONTROL WITH TIME DELAY}

Our goal is to stabilize the rectilinear motion along the $x$ axis. First, this is achieved by generating the steering angle using the delayed feedback of the vehicle's lateral position and yaw angle:

$$
\delta_{\mathrm{S}}(t)=-P_{y} y(t-\tau)-P_{\psi} \psi(t-\tau)
$$

where $\tau$ denotes the total delay in the system, including measurement and communication delays, processing time, and the dynamics of the actuators and the steering mechanism. $P_{y}$ and $P_{\psi}$ denote the proportional gains of the vehicle's lateral position and yaw angle. We are going to refer to this control method in the following as the PP controller.

After substituting the control law Eqn. (18) into the equations of motion of the dynamic vehicle model, the system is linearized about the rectilinear motion along the $y=0$ line. This leads to the state space representation

$$
\dot{\mathbf{x}}(t)=\mathbf{A x}(t)+\mathbf{B u}(t-\tau)
$$

where $\mathbf{u}(t)=-P_{y} y(t)-P_{\psi} \psi(t)$. Since the longitudinal position $x$ does not influence stability as it can be decoupled from the other equations, the state vector can be reduced to $\mathbf{x}=\left[\begin{array}{llll}y & \psi & \sigma_{1} & \sigma_{2}\end{array}\right]^{\mathrm{T}}$. Consequently, the system and input matrices are

$$
\mathbf{A}=\left[\begin{array}{cccc}
0 & V & 1 & 0 \\
0 & 0 & 0 & 1 \\
0 & 0 & A_{33} & A_{34} \\
0 & 0 & A_{43} & A_{44}
\end{array}\right], \quad \mathbf{B}=\left[\begin{array}{c}
0 \\
0 \\
B_{3} \\
B_{4}
\end{array}\right]
$$

with elements

$$
\begin{gathered}
A_{33}=-\frac{B_{3}}{V}-\frac{C_{\mathrm{R}}\left(d^{2} m+J_{z}\right)}{m V J_{z}}, \quad A_{34}=-B_{3} \frac{f}{V}-V, \\
A_{43}=-\frac{B_{4}}{V}+\frac{C_{\mathrm{R}} d}{V J_{z}}, \quad A_{44}=-B_{4} \frac{f}{V},
\end{gathered}
$$

and

$$
B_{3}=\frac{C_{\mathrm{F}}\left(d(d-f) m+J_{z}\right)}{m J_{z}}, \quad B_{4}=\frac{C_{\mathrm{F}}(f-d)}{J_{z}}
$$

Using the gain vector $\mathbf{K}=\left[\begin{array}{llll}-P_{y}-P_{\psi} & 0 & 0\end{array}\right]$, the steering angle can be written as $\mathbf{u}(t-\tau)=\mathbf{K} \mathbf{x}(t-\tau)$, leading to the characteristic equation

$$
D(\lambda):=\operatorname{det}\left(\lambda \mathbf{I}-\mathbf{A}-\mathbf{B K} \mathrm{e}^{-\lambda \tau}\right)=0,
$$

where $\lambda \in \mathbb{C}$ is the characteristic exponent and I denotes the identity matrix. The characteristic function $D(\lambda)$ is of the form

$$
\lambda^{4}+a_{3} \lambda^{3}+\left(a_{2, a}+a_{2, b} \mathrm{e}^{-\lambda \tau}\right) \lambda^{2}+a_{1} \mathrm{e}^{-\lambda \tau} \lambda+a_{0} \mathrm{e}^{-\lambda \tau}
$$

with coefficients

$$
\begin{gathered}
a_{3}=\frac{C_{\mathrm{F}}\left(d^{2} m-2 d f m+f^{2} m+J_{z}\right)+C_{\mathrm{R}}\left(d^{2} m+J_{z}\right)}{J_{z} m V}, \\
a_{2, a}=\frac{m V^{2}\left(d\left(C_{\mathrm{F}}+C_{\mathrm{R}}\right)-C_{\mathrm{F}} f\right)+C_{\mathrm{F}} C_{\mathrm{R}} f^{2}}{J_{z} m V^{2}} \\
a_{2, b}=\frac{C_{\mathrm{F}} V^{2}\left(m(d-f)\left(d P_{y}-P_{\psi}\right)+J_{z} P_{y}\right)}{J_{z} m V^{2}} \\
a_{1}=\frac{C_{\mathrm{F}} C_{\mathrm{R}} f P_{\psi}}{J_{z} m V}, \quad a_{0}=\frac{C_{\mathrm{F}} C_{\mathrm{R}} f P_{y}}{J_{z} m}
\end{gathered}
$$

In the delay-free case ( $\tau=0$ ), Eqn. (24) reduces to a polynomial, with the second degree coefficient $a_{2}=a_{2, a}+a_{2, b}$. The stability analysis in this case can be performed using the Routh-Hurwitz 
criterion. The delay-free system is stable, if the polynomial coefficients $a_{0}, a_{1}, a_{2}$ and $a_{3}$, as well as the following Hurwitz determinant are all positive:

$$
\Delta_{3}=\left|\begin{array}{ccc}
a_{3} & a_{1} & 0 \\
a_{4} & a_{2} & a_{0} \\
0 & a_{3} & a_{1}
\end{array}\right| .
$$

The corresponding boundaries of stability and the stable domain in the $\left(P_{y}, P_{\psi}\right)$ plane is depicted in Fig. 2 (a). Note that the coefficient $a_{3}$ depends only on the physical vehicle parameters and, assuming that all of them (including $V$ ) are positive, it will always be positive. Along the boundary $a_{0}=0$, a real characteristic exponent crosses the imaginary axis, leading to a static loss of stability. This translates to $P_{y}=0$ in the $\left(P_{y}, P_{\psi}\right)$ plane. On the other hand, the $\Delta_{3}=0$ curve represents the boundary of dynamic stability loss (Hopf bifurcation).

When $\tau>0$, the D-subdivision method is used to determine the stable domains. This involves substituting $\lambda=\mathrm{i} \omega$ into the characteristic equation, then separating the real and imaginary parts. The resulting equations can be solved for $P_{y}$ and $P_{\psi}$, leading to a parametric expression of the so-called D-curves as a function of $\omega$ (the angular frequency of the resulting oscillatory motion at a given point of the D-curves). Similarly to the delayfree case, the boundary of static stability loss is $P_{y}=0$. The number of unstable characteristic roots in the different regions of the parameter plane can be calculated using Stepan's formulae [26]. The region where this number is zero is going to be the stable domain. Fig. 2 (b) shows how the stable region shrinks with increasing time delay.
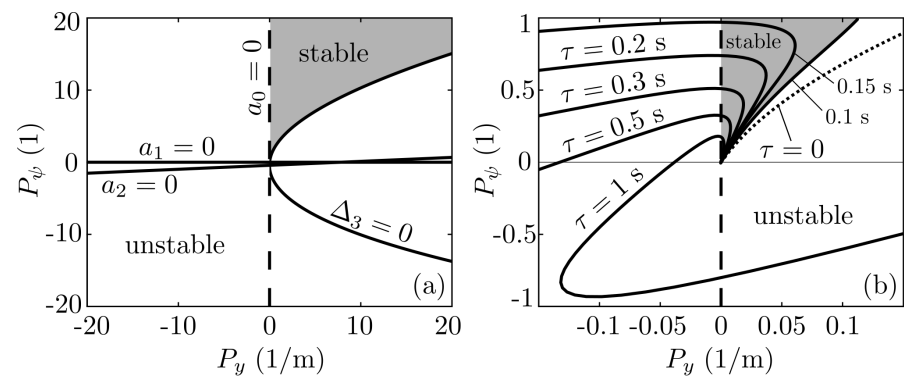

FIGURE 2. Stability charts of the dynamic vehicle model with simple state feedback with (a) zero feedback delay and (b) non-zero feedback delay (the dotted line shows the $\Delta_{3}=0$ boundary of the delay-free system for comparison and the gray shading corresponds to the stable region for $\tau=0.1 \mathrm{~s}$ ). Dashed lines represent the boundaries of static loss of stability.

\section{FINITE SPECTRUM ASSIGNMENT}

In order to overcome the negative effects of feedback delay, we are going to employ a predictor based control approach, called finite spectrum assignment $[13,14]$. The main idea of predictor control is to use a mathematical model of the plant to calculate a prediction for the current state values based on the delayed feedback information. These predicted states are then used to determine the necessary control action.

\section{Theoretical Background}

The FSA controller considers a linear state space model of the plant as the predictive model:

$$
\dot{\mathbf{x}}(t)=\widetilde{\mathbf{A}} \mathbf{x}(t)+\widetilde{\mathbf{B}} \mathbf{u}(t-\tilde{\tau}) .
$$

The parameters of the predictive model within the controller are going to be denoted by tildes. If the (assumed) time delay of the system is $\tilde{\tau}$, then the FSA controller calculates the solution of Eqn. (26) over an interval of $\tilde{\tau}$ by taking $\mathbf{x}(t-\tilde{\tau})$ as initial condition. This leads to the control law

$$
\mathbf{u}(t)=\mathbf{K} \mathrm{e}^{\widetilde{\mathbf{A}} \tilde{\tau}} \mathbf{x}(t)+\mathbf{K} \int_{-\tilde{\tau}}^{0} \mathrm{e}^{-\widetilde{\mathbf{A} s} \widetilde{\mathbf{B}} \mathbf{u}(t+s) \mathrm{d} s .}
$$

It can be shown that if the internal model in Eqn. (26) perfectly matches the real system $(\widetilde{\mathbf{A}}=\mathbf{A}, \widetilde{\mathbf{B}}=\mathbf{B}$ and $\tilde{\tau}=\tau)$, then the above control law cancels out the delayed terms, leading to the simplified dynamics $\dot{\mathbf{x}}(t)=(\mathbf{A}+\mathbf{B K}) \mathbf{x}(t)$. This reduces the infinite dimensional nature of the original delayed problem to a finite number of poles that can be freely assigned through $\mathbf{K}$, given that the pair $\mathbf{A}$ and $\mathbf{B}$ is controllable.

However, in practice, the control law Eqn. (27) can hardly be implemented accurately. On one hand, because of parameter uncertainties and modeling errors, the internal model will almost always be different from the controlled system. As a result, the delayed terms cannot be canceled out and the problem will remain infinite dimensional. Thus arbitrary pole placement cannot be achieved in this case.

On the other hand, the integral term in the control law can only be approximated in practice, which may also lead to stability issues. Using numerical quadrature to calculate the integral term modifies the control law to

$$
\mathbf{u}(t)=\mathbf{K} \mathrm{e}^{\widetilde{\mathbf{A}} \tilde{\tau}} \mathbf{x}(t)+\mathbf{K} \sum_{j=0}^{\tilde{r}} \mathrm{e}^{\widetilde{\mathbf{A}} \theta_{j, \tilde{r}} \widetilde{\mathbf{B}} \mathbf{u}\left(t-\theta_{j, \tilde{r}}\right) h_{j, \tilde{r}}}
$$

where $\theta_{j, \tilde{r}} \in[0, \tilde{\tau}], h_{j, \tilde{r}} \in \mathbb{R}$, and $\tilde{r}$ is an integer that determines the precision of the approximation: $\tilde{r} \rightarrow \infty$ produces the exact value of the integral. This way the distributed time delay in the 
original control law is approximated by a sum of point delays and the resulting system is defined by a set of neutral functional differential equations:

$$
\begin{gathered}
\dot{\mathbf{x}}(t)=\mathbf{A} \mathbf{x}(t)+\mathbf{B u}(t-\tau) \\
\dot{\mathbf{u}}(t)=\mathbf{K e}^{\widetilde{\mathbf{A}} \tilde{\tau}} \mathbf{A} \mathbf{x}(t)+\mathbf{K} \mathrm{e}^{\widetilde{\mathbf{A}} \tilde{\tau}} \mathbf{B} \mathbf{u}(t-\tau) \\
+\sum_{j=0}^{\tilde{r}} \mathbf{K} \mathrm{e}^{\widetilde{\mathbf{A}} \theta_{j, r}} \widetilde{\mathbf{B}} \dot{\mathbf{u}}\left(t-\theta_{j, \tilde{r}}\right) h_{j, \tilde{r}}
\end{gathered}
$$

This neutral system, however, may become unstable even though the original system was stable, regardless of the resolution of the numerical quadrature $[27,28]$. This is explained by the appearance of a set of unstable characteristic roots with a large modulus when the numerical approximation is introduced. As $\tilde{r}$ is increased, some of these roots tend to the roots of the original system, but there may be others that move off to infinity without leaving the right half plane.

It was shown in [27], that a necessary condition for the stability of the closed loop system with control law Eqn. (28) is the stability of the difference part of Eqn. (29) and Eqn. (30), i.e.

$$
\begin{aligned}
\mathbf{x}(t) & =\mathbf{0}, \\
\mathbf{u}(t) & =\sum_{j=0}^{\tilde{r}} \mathbf{K} \mathrm{e}^{\widetilde{\mathbf{A}} \theta_{j, \tilde{r}}} \widetilde{\mathbf{B}} \mathbf{u}\left(t-\theta_{j, \tilde{r}}\right) h_{j, \tilde{r}}
\end{aligned}
$$

As $\tilde{r} \rightarrow \infty$, this latter condition is equivalent to the stability of

$$
\mathbf{u}(t)=\mathbf{K} \int_{-\tilde{\tau}}^{0} \mathrm{e}^{-\widetilde{\mathbf{A}} s} \widetilde{\mathbf{B}} \mathbf{u}(t+s) \mathrm{d} s
$$

Finally, it was also shown in [29] that small perturbations of the integral time step may also lead to unstable behavior, which is important regarding the choice of integration scheme. In the single input case, the corresponding stability criterion is

$$
S=\int_{0}^{\tilde{\tau}}\left|\mathbf{K e}^{\widetilde{\mathbf{A}} s} \widetilde{\mathbf{B}}\right| \mathrm{d} s<1 .
$$

Following [28], we are going to refer to the case when the original closed loop system with an accurate implementation of the integral is stable as ideal stability. If the functional differential equation in Eqn. (33) is also stable, we refer to it as theoretical stability. Additionally, if the condition in Eqn. (34) regarding robustness to perturbations of the integral time step is also met, it will be referred to as robust stability.

Since the conditions of theoretical and robust stability are related to unstable characteristic roots with large imaginary parts, these restrictions can be avoided by applying a low-pass filter or piece-wise constant input through a digital controller. See [30] for a more detailed examination of these restrictions.

\section{Application to the Vehicle Model}

We are going to use the linearized version of the kinematic vehicle model as the predictive model of the FSA controller. The corresponding system and input matrices are

$$
\widetilde{\mathbf{A}}=\left[\begin{array}{ll}
0 & \tilde{V} \\
0 & 0
\end{array}\right], \quad \widetilde{\mathbf{B}}=\left[\begin{array}{c}
0 \\
\tilde{V} / \tilde{f}
\end{array}\right],
$$

leading to the steering angle definition (see Eqn. (27))

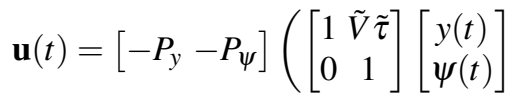

$$
\begin{aligned}
& \left.+\int_{-\tilde{\tau}}^{0}\left[\begin{array}{cc}
1 & -s \tilde{V} \\
0 & 1
\end{array}\right]\left[\begin{array}{c}
0 \\
\tilde{V} / \tilde{f}
\end{array}\right] \mathbf{u}(t+s) \mathrm{d} s\right) .
\end{aligned}
$$

Applying the above control law to the linearized dynamic vehicle model and assuming the solution to be in the exponential form $\mathbf{C e}^{\lambda t}\left(\mathbf{C} \in \mathbb{C}^{5}\right)$, the system can be written as

$$
\underbrace{\left[\begin{array}{ccccc}
\lambda & -V & -1 & 0 & 0 \\
0 & \lambda & 0 & -1 & 0 \\
0 & 0 & \lambda-A_{33} & -A_{34} & -B_{3} \mathrm{e}^{-\lambda \tau} \\
0 & 0 & -A_{43} & \lambda-A_{44} & -B_{4} \mathrm{e}^{-\lambda \tau} \\
P_{y} P_{\psi}+P_{y} \tilde{V} \tilde{\tau} & 0 & 0 & g(\lambda)
\end{array}\right]}_{\mathbf{M}(\lambda)} \mathbf{C}=\mathbf{0},
$$

where $\mathbf{M}(\lambda)$ includes the elements of $\mathbf{A}$ and $\mathbf{B}$ from Eqn. (20), and

$$
\begin{aligned}
g(\lambda) & =\frac{1}{\tilde{f} \lambda^{2}}\left(\tilde{f} \lambda^{2}-\tilde{V} \mathrm{e}^{-\lambda \tilde{\tau}}\left(P_{y}(\lambda \tilde{\tau} \tilde{V}+\tilde{V})+\lambda P_{\psi}\right)\right. \\
& \left.+P_{y} \tilde{V}^{2}+\lambda P_{\psi} \tilde{V}\right)
\end{aligned}
$$

This leads to the characteristic equation of the system as

$$
D(\lambda):=\operatorname{det}(\mathbf{M}(\lambda))=0
$$

from which the boundaries of ideal stability can be determined using D-subdivision. This stability condition applies if the integral term in the FSA control law can be implemented accurately. If numerical approximation is used, then the functional difference equation in Eqn. (33) must also be stable in order to 
guarantee stability of the closed loop system. The characteristic equation of Eqn. (33) can be reached by substituting $\mathbf{x}(t) \equiv \mathbf{0}$ and $\delta_{\mathrm{S}}(t)=\delta_{\mathrm{s}, 0} \mathrm{e}^{\lambda t}$ into the control law:

$$
\begin{aligned}
D(\lambda) & =\frac{1}{\tilde{f} \lambda^{2}}\left(\left(\lambda\left(\tilde{f} \lambda+P_{\psi} \tilde{V}\right)+P_{y} \tilde{V}^{2}\right)+\right. \\
& \left.-\mathrm{e}^{-\lambda \tilde{\tau}} \tilde{V}\left(P_{y}(\lambda \tilde{\tau} \tilde{V}+\tilde{V})+\lambda P_{\psi}\right)\right) .
\end{aligned}
$$

Finally, the condition of robust stability with regards to perturbations of the integral time step is

$$
S=\int_{0}^{\tilde{\tau}}\left|\frac{\tilde{V}\left(-P_{y} s \tilde{V}-P_{\psi}\right)}{\tilde{f}}\right| \mathrm{d} s<1
$$
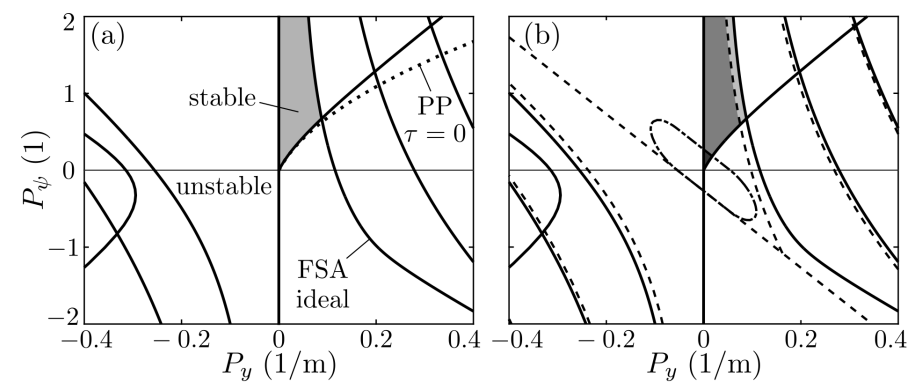

FIGURE 3. Application of the FSA controller (based on the kinematic vehicle model) to the dynamic model for $V=20 \mathrm{~m} / \mathrm{s}$ and $\tau=0.5 \mathrm{~s}$. Panel (a): stability chart of ideal stability (solid lines and shading). The dotted line represents the border of stability of the delay-free PP controller. Panel (b): stability chart showing the superposition of ideal (solid lines), theoretical (dashed) and robust stability (dash-dot line).

Figure 3 (a) shows the stable region of ideal stability. Although the parameter values of the internal model match those of the dynamic vehicle model $(\tilde{f}=f, \tilde{V}=V, \tilde{\tau}=\tau)$, since not all of the dynamics are included in the controller, the stable region is significantly smaller than in the case of the delay-free PP controller. It is also interesting to note that parts of the stability boundary of the FSA controller are very close to the boundaries of theoretical stability (Fig. 3 (b)). This is also because the dynamics of the internal model differ from the vehicle model, and thus, the FSA controller cannot completely cancel out the delayed terms, even when there are no parameter mismatches. This means that the effects of the controller always remain present, and we cannot get back the stability regions of the delay free PP controller even with perfect implementation and zero parameter errors. However, if we used the linearized dynamic model within the controller, then (assuming no parameter errors and perfect
TABLE 1. Vehicle Parameters Used in Stability Maps and Simulations

\begin{tabular}{lll}
\hline Parameter & Notation & Value \\
\hline Vehicle wheelbase & $f$ & $2.7 \mathrm{~m}$ \\
$\begin{array}{l}\text { Distance between rear } \\
\text { axle and center of gravity }\end{array}$ & $d$ & $1.35 \mathrm{~m}$ \\
Vehicle mass & $m$ & $1430 \mathrm{~kg}$ \\
Yaw moment of inertia & $J_{z}$ & $2500 \mathrm{kgm}^{2}$ \\
Lateral stiffness of front tire & $C_{\mathrm{F}}$ & $45 \mathrm{kN}$ \\
Lateral stiffness of rear tire & $C_{\mathrm{R}}$ & $45 \mathrm{kN}$ \\
Longitudinal velocity & $V$ & $20 \mathrm{~m} / \mathrm{s}$ \\
Time delay & $\tau$ & $0.5 \mathrm{~s}$ \\
\hline
\end{tabular}

implementation) the characteristic equation would reduce to that of the delay-free PP controller, and the full stable region of Fig. 2 (a) would indeed be stable for the FSA controller too.

\section{NUMERICAL SIMULATIONS}

The vehicle parameters used for the numerical simulations are listed in Table 1 (the same parameters were used for the creation of stability maps too). Using the semi-discretization method [31], we determined the control gains that lead to the most highly damped system response: for the PP controller, these are $P_{y}=0.00077 \mathrm{~m}^{-1}$ and $P_{\psi}=0.0805$, while for the FSA $P_{y}=0.0016 \mathrm{~m}^{-1}$ and $P_{\psi}=0.1253$. This means that the characteristic multipliers of the corresponding semi-discrete systems were closest to zero at these parameter values. The simulations were run using the nonlinear vehicle model. An initial position of $y(0)=3.75 \mathrm{~m}$ and $\psi(0)=0$ was selected to model a lane change maneuver. For the time interval $t \in[-\tau, 0)$, all initial values were set to zero, representing that a change in the reference signal (i.e. the decision to perform a lane change) only occurs at $t=0$. Therefore, because of the time delay, the FSA controller assumes in the first $\tau$ time interval of the simulations that the vehicle is on the desired path, and no control action is needed.

\section{Theoretical and Robust Stability}

The simulation results in Fig. 4 demonstrate the significance of theoretical and robust stability. A rectangular approximation of the integral term was used within the FSA controller, with a nominal time step of $0.025 \mathrm{~s}$. The simulation time step was set to $0.0025 \mathrm{~s}$. The integration time step, however, was periodically varied according to the following rule: $\Delta t_{1}=0.025 \mathrm{~s}$, $\Delta t_{2}=1.5 \Delta t_{1}, \Delta t_{3}=\Delta t_{1}$ and $\Delta t_{4}=0.5 \Delta t_{1}$. As a result, the control gains have to be chosen from the robustly stable area in order to achieve stability (Fig. 4 (a)). If a uniform integration time 


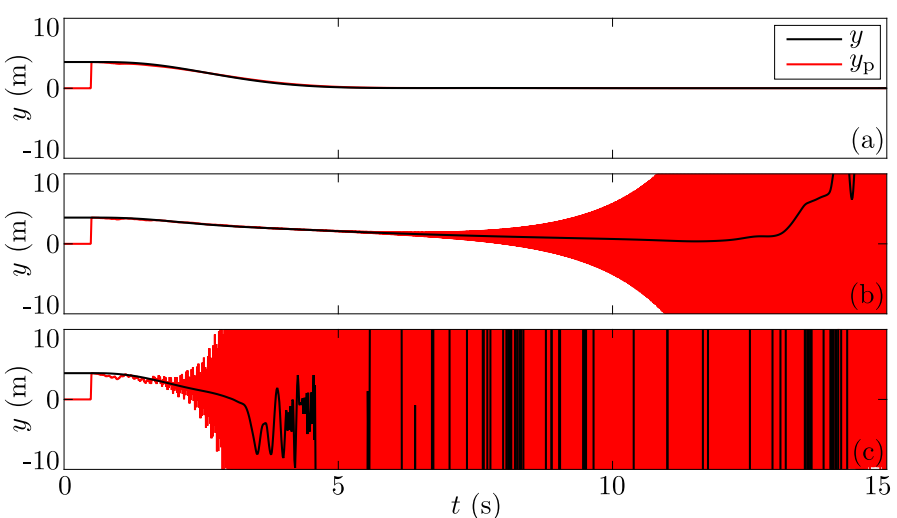

FIGURE 4. Actual (black) and predicted (red) system response of the vehicle model with FSA using a rectangular approximation of the integral with periodically varied time steps. The control parameters are chosen such that (a) robust stability is ensured $\left(P_{y}=0.0048 \mathrm{~m}^{-1}\right.$, $\left.P_{\psi}=0.237\right)$, (b) the system is theoretically stable, but not robustly stable $\left(P_{y}=0.01 \mathrm{~m}^{-1}, P_{\psi}=1.2\right)$, (c) only ideal stability is ensured $\left(P_{y}=0.04 \mathrm{~m}^{-1}, P_{\psi}=1.6\right)$.

step is used, however, the theoretically (but not robustly) stable domain will also lead to stable behavior. Otherwise, as shown in Fig. 4 (b), the continuously increasing oscillations of the predicted states will eventually lead the system to lose its stability. Parameter pairs from the ideally, but not theoretically stable domain can only stabilize the system if the integration time step and the simulation time step are the same, which would correspond to a discretized approximation of a continuous implementation of the integral. Otherwise, as in Fig. 4 (c), the system will quickly lose its stability. Note, however, that the exact stability boundaries also depend on the details of implementation (simulation and integration time steps, and the kind of perturbation added), therefore they are generally different from the analytically determined results. The exact stable regions can be checked for example by semi-discretization [31].

Since the instability mechanisms of theoretical and robust stability are related to higher frequency ranges, the use of a digital controller will overcome these restrictions. Assuming that the automotive implementation in our example would be digital anyways, the following analysis will be limited to ideal stability. Nevertheless, we deemed it important to be aware of the previously detailed mechanisms.

\section{Parameter Mismatches}

Figure 5 shows how parameter errors in terms of $V$ and $\tau$ affect the (ideally) stable regions of the FSA controller. Thus, not only does the internal model differ from the controlled vehicle by not including tire dynamics, but the velocity and time delay values used for prediction are also off in these cases. The sta-

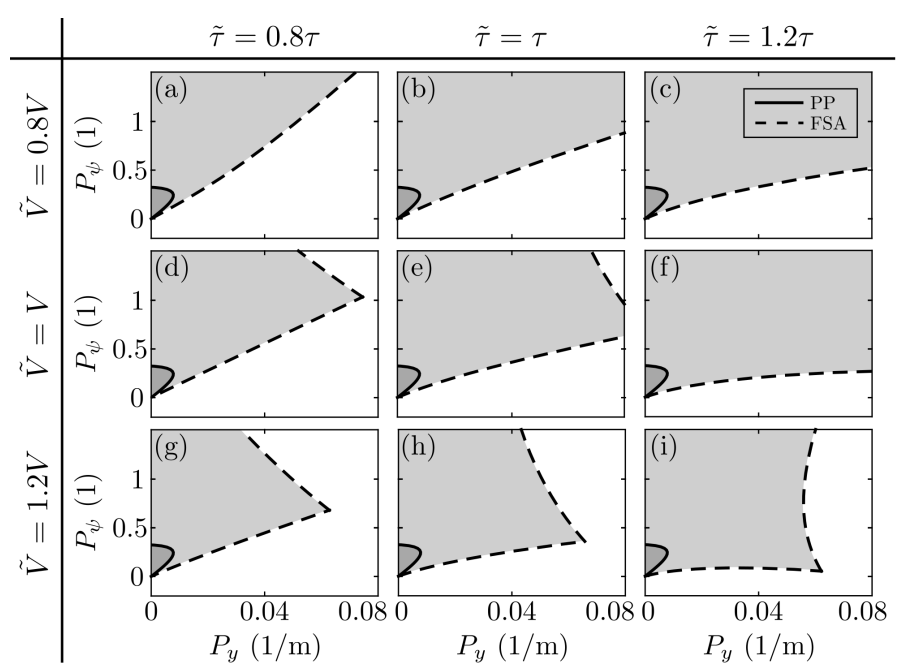

FIGURE 5. Stable regions of the dynamic vehicle model with the PP controller (solid borders) and the FSA controller (dashed) in case of $-20 \%, 0 \%$ and $+20 \%$ errors in the estimated vehicle velocity and/or the time delay $(V=20 \mathrm{~m} / \mathrm{s}$ and $\tau=0.5 \mathrm{~s})$.

bility regions of the delayed feedback controller are also plotted for comparison. Numerical simulations were also run for the 9 cases of Fig. 5, all using the optimal control gains for case (e), i.e. no parameter mismatches are assumed while tuning the controller. The time histories of the simulations are shown in Fig. 6, including the trajectories predicted by the FSA controller.

Settling time was calculated to measure the performance of the two controllers. We define settling time as the lowest time instant $t^{*}$ for which $|y(t)|<0.02|y(0)| \forall t>t^{*}$. In addition, the root mean square error (RMSE) between the predicted and actual system states was chosen to measure prediction accuracy for the FSA controller:

$$
\operatorname{RMSE}_{y}=\sqrt{\frac{1}{N} \sum_{i=1}^{N}\left(y_{i}-y_{\mathrm{p}, i}\right)^{2}},
$$

where $y_{i}$ and $y_{\mathrm{p}, i}$ are the actual and predicted state values at the $i$ th simulation time step. In order to accentuate the differences between the investigated cases, the first $\tau$ time interval, where all predictions have the same error in $y$ (with magnitude of $|y(0)|$ ), was not included in the above sum. The errors were considered until $t=10 \mathrm{~s}$ and $\mathrm{RMSE}_{\psi}$ was calculated the same way. The actual values of settling time and RMSE are listed in Table 2 .

The stable regions of the FSA controller are significantly larger than those of the PP controller, even when the velocity and time delay values used in the internal model have a $20 \%$ error (the third parameter of the internal model, the wheelbase $f$ is assumed to be known accurately). In fact, the stable area of the PP controller is only comparable in size to the analytically deter- 


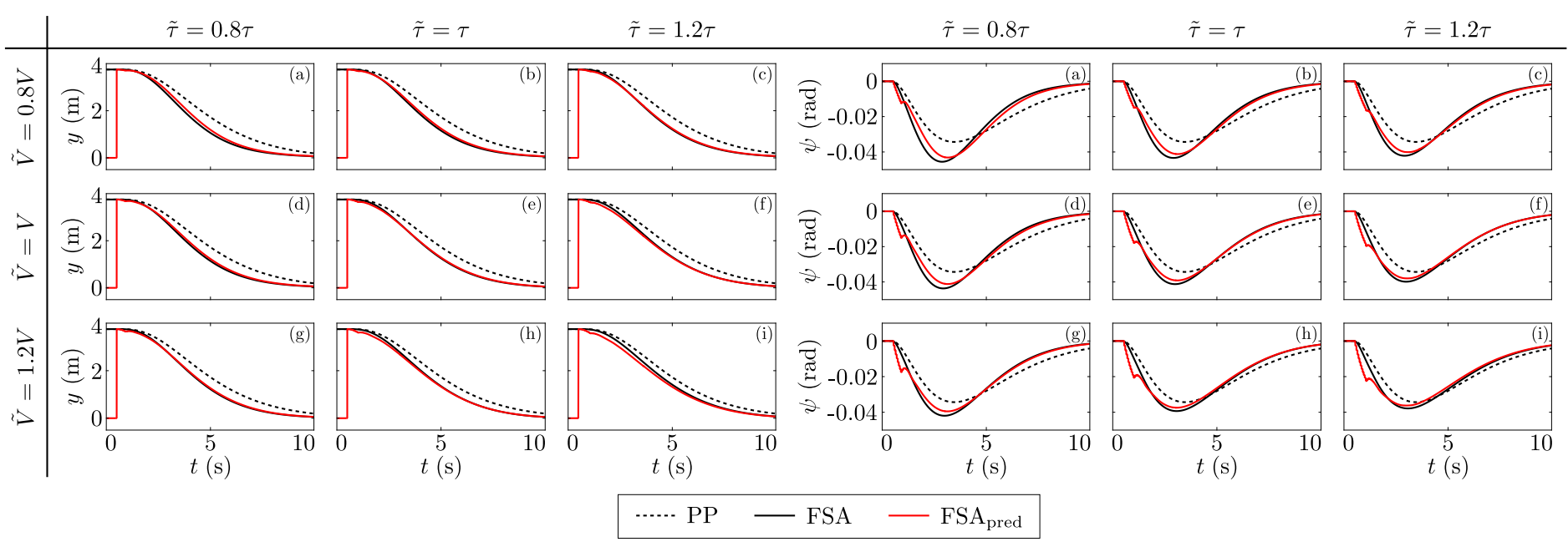

FIGURE 6. Simulation results of the PP controller (dotted lines) and the FSA controller (solid lines) in case of $-20 \%, 0 \%$ and $+20 \%$ errors in the estimated vehicle velocity and/or the time delay $(V=20 \mathrm{~m} / \mathrm{s}$ and $\tau=0.5 \mathrm{~s})$. The predicted trajectories of the FSA controller are also shown in red.

mined robustly stable domain of the FSA controller (the darkest shaded area in Fig. 3 (b)). The optimal point of this larger stable domain is located at higher control gains, which leads to a faster system response. The settling time values in Table 2 show that even in the worst case, when both the vehicle velocity and the time delay are overestimated (case (i)), the FSA steers the vehicle $\sim 15 \%$ faster into the desired lane than the PP controller. The RMSE of the predicted lateral vehicle position is also the largest in this case, but it is still less than $11 \mathrm{cms}$ (which is approximately $3 \%$ of the initial position).

Since the dynamics of the steering mechanism are not modeled, as the FSA controller becomes aware of the change in reference at $t=\tau$, the steering angle jumps instantaneously to the value determined by the controller. According to the kinematic model, the vehicle can directly follow this non-smooth jump in the steering angle, thus the predicted vehicle heading of the FSA controller includes a kink at $t=\tau$ (see Fig. 6). Because of the elasticity of the tires, however, the vehicle initially follows a larger turning radius than predicted. At $t=2 \tau$ the result of the initial steering action reaches the controller, which leads to another non-smooth change in the predicted signals and consequently in the steering angle. The elastic tires, however, minimize the effect of these sudden changes of the steering angle on the actual vehicle path.

\section{CONCLUSION}

The application of the predictor control approach called finite spectrum assignment was demonstrated in this paper for lateral vehicle control. The model used for predictions was a simplified version of the controlled system, which lead to imperfect predictions even in the so-called ideal case. Nevertheless,
TABLE 2. Settling Time and Root Mean Square Error Values of the Dynamic Vehicle Model

\begin{tabular}{cccccc}
\hline & \multicolumn{2}{c}{ Settling Time $(\mathrm{s})$} & & \multicolumn{2}{c}{ RMSE FSA } \\
\cline { 2 - 3 } \cline { 5 - 6 } Case & PP & FSA & & $y(\mathrm{~m})$ & $\psi(\mathrm{rad})$ \\
\hline (a) & 11.799 & 9.709 & & 0.092 & 0.0028 \\
(b) & 11.799 & 9.553 & & 0.056 & 0.0019 \\
(c) & 11.799 & 9.851 & & 0.031 & 0.0016 \\
(d) & 11.799 & 9.559 & & 0.057 & 0.0022 \\
(e) & 11.799 & 9.512 & & 0.035 & 0.0018 \\
(f) & 11.799 & 9.891 & & 0.059 & 0.0019 \\
(g) & 11.799 & 9.500 & & 0.036 & 0.0022 \\
(h) & 11.799 & 9.559 & & 0.062 & 0.0023 \\
(i) & 11.799 & 10.006 & & 0.109 & 0.0026 \\
\hline
\end{tabular}

it was shown that even in the presence of parameter mismatches on top of the modeling simplifications, the FSA controller is able to steer the vehicle into the desired trajectory significantly faster than simple delayed state feedback. This, however, comes at the price of increased computational requirements.

Future work may involve testing the limits of the system in more challenging scenarios, such as higher speeds, varying path curvature, etc., where the differences between the two vehicle models are more pronounced.

\section{ACKNOWLEDGMENT}

This research was partly supported by the National Research, Development and Innovation Office under grant no. 
NKFI-128422 and by the Higher Education Excellence Program of the Ministry of Human Capacities in the frame of Artificial intelligence research area of Budapest University of Technology and Economics (BME FIKP-MI).

\section{REFERENCES}

[1] Amer, N. H., Zamzuri, H., Hudha, K., and Kadir, Z. A., 2017. "Modelling and control strategies in path tracking control for autonomous ground vehicles: a review of state of the art and challenges". Journal of Intelligent \& Robotic Systems, 86(2), pp. 225-254.

[2] Amidi, O., and Thorpe, C. E., 1991. "Integrated mobile robot control". In Mobile Robots V, Vol. 1388, International Society for Optics and Photonics, pp. 504-524.

[3] Hoffmann, G. M., Tomlin, C. J., Montemerlo, M., and Thrun, S., 2007. "Autonomous automobile trajectory tracking for off-road driving: Controller design, experimental validation and racing". In American Control Conference, 2007. ACC'07, IEEE, pp. 2296-2301.

[4] Fierro, R., and Lewis, F. L., 1997. "Control of a nonholomic mobile robot: Backstepping kinematics into dynamics". Journal of robotic systems, 14(3), pp. 149-163.

[5] Sharp, R., Casanova, D., and Symonds, P., 2000. "A mathematical model for driver steering control, with design, tuning and performance results". Vehicle system dynamics, 33(5), pp. 289-326.

[6] Falcone, P., Borrelli, F., Asgari, J., Tseng, H. E., and Hrovat, D., 2007. "Predictive active steering control for autonomous vehicle systems". IEEE Transactions on control systems technology, 15(3), pp. 566-580.

[7] Heredia, G., and Ollero, A., 2007. "Stability of autonomous vehicle path tracking with pure delays in the control loop". Advanced Robotics, 21(1-2), pp. 23-50.

[8] Liu, Q., Liu, Y., Liu, C., Chen, B., Zhang, W., Li, L., and Ji, X., 2018. "Hierarchical lateral control scheme for autonomous vehicle with uneven time delays induced by vision sensors". Sensors, 18(8), p. 2544.

[9] Wang, Y., Nguyen, B. M., Fujimoto, H., and Hori, Y., 2013. "Vision-based integrated lateral control system for electric vehicles considering multi-rate and measurable uneven time delay issues". In 2013 IEEE International Symposium on Industrial Electronics, IEEE, pp. 1-6.

[10] Kosecka, J., 1996. "Vision-based lateral control of vehicles: Look-ahead and delay issues".

[11] Hsu, J.-C., and Tomizuka, M., 1998. "Analyses of visionbased lateral control for automated highway system". Vehicle system dynamics, 30(5), pp. 345-373.

[12] Mobus, R., and Zomotor, Z., 2005. "Constrained optimal control for lateral vehicle guidance". In IEEE Proceedings. Intelligent Vehicles Symposium, 2005., IEEE, pp. 429434.
[13] Manitius, A. Z., and Olbrot, A. W., 1979. "Finite spectrum assignment problem for systems with delays". IEEE Transactions on Automatic Control, 24(4), pp. 541-553.

[14] Wang, Q.-G., Tong, H. L., and Kok, K. T., 1999. FiniteSpectrum Assignment for Time-Delay Systems. SpringerVerlag London.

[15] Molnár, T., and Insperger, T., 2016. "On the robust stabilizability of unstable systems with feedback delay by finite spectrum assignment". Journal of Vibration and Control, 22(3), pp. 649-661.

[16] Vörös, I., and Várszegi, B., 2018. "Lateral vehicle control using finite spectrum assignment". IFAC-PapersOnLine, 51(14), pp. 306-311.

[17] Pacejka, H. B., 2006. Tyre and Vehicle Dynamics. Butterworth-Heinemann, Oxford.

[18] Rajamani, R., 2006. Vehicle Dynamics and Control. Springer.

[19] Bloch, A., 2003. Nonholonomic mechanics and control, Vol. 24. Springer, New York, NY, USA.

[20] Gantmacher, F., 1975. Lectures in Analytical Mechanics. MIR Publiser, Moscow.

[21] Baruh, H., 1999. Analytical Dynamics. WCB/McGrawHill, Boston, MA, USA.

[22] Greenwood, D. T., 2006. Advanced Dynamics. Cambridge University Press.

[23] Kane, T. R., and Levinson, D. A., 2005. Dynamics, theory and applications. Internet-First University Press., Ithaca, NY, USA.

[24] Takács, D., and Stépán, G., 2013. “Contact patch memory of tyres leading to lateral vibrations of four-wheeled vehicles". Phil. Trans. R. Soc. A, 371(1993), p. 20120427.

[25] Várszegi, B., Takács, D., Stépán, G., and Hogan, S. J., 2016. "Stabilizing skateboard speed-wobble with reflex delay". Journal of The Royal Society Interface, 13(121).

[26] Stépán, G., 1989. Retarded Dynamical Systems: Stability and Characteristic Functions. Longman Scientific \& Technical.

[27] Mondié, S., Dambrine, M., and Santos, O., 2002. "Approximation of control laws with distributed delays: a necessary condition for stability". Kybernetika, 38(5), pp. 541-551.

[28] Michiels, W., and Niculescu, S.-I., 2007. Stability and Stabilization of Time-Delay Systems. Society for Industrial \& Applied Mathematics, U.S.

[29] Michiels, W., Mondié, S., and Roose, D., 2003. Robust stabilization of time-delay systems with distributed delay control laws: Necessary and sufficient conditions for a safe implementation. Tech. rep., Katholieke Universiteit Leuven, Belgium.

[30] Zhong, Q. C., 2006. Robust control of time-delay systems. Springer.

[31] Insperger, T., and Stépán, G., 2011. Semi-Discretization for Time-Delay Systems. Springer. 